\title{
Operations on Well-Covered Graphs and the Roller-Coaster Conjecture
}

\author{
Philip Matchett \\ Emmanuel College \\ Cambridge CB2 3AP \\ ENGLAND \\ pmatchet@d.umn.edu
}

Submitted: Feb 29, 2004; Accepted: Jun 15, 2004; Published: Jul 1, 2004

MR Subject Classifications: 05C69, 05C75

\begin{abstract}
A graph $G$ is well-covered if every maximal independent set has the same cardinality. Let $s_{k}$ denote the number of independent sets of cardinality $k$, and define the independence polynomial of $G$ to be $S(G, z)=\sum s_{k} z^{k}$. This paper develops a new graph theoretic operation called power magnification that preserves well-coveredness and has the effect of multiplying an independence polynomial by $z^{c}$ where $c$ is a positive integer. We will apply power magnification to the recent Roller-Coaster Conjecture of Michael and Traves, proving in our main theorem that for sufficiently large independence number $\alpha$, it is possible to find well-covered graphs with the last $(.17) \alpha$ terms of the independence sequence in any given linear order. Also, we will give a simple proof of a result due to Alavi, Malde, Schwenk, and Erdős on possible linear orderings of the independence sequence of not-necessarily well-covered graphs, and we will prove the Roller-Coaster Conjecture in full for independence number $\alpha \leq 11$. Finally, we will develop two new graph operations that preserve well-coveredness and have interesting effects on the independence polynomial.
\end{abstract}

\section{Introduction}

Let $G=(V, E)$ be a graph without loops or multiple edges. A set of vertices $W \subset V$ is called independent if no two vertices in $W$ are adjacent. The independence number of $G$ is the cardinality of the largest independent set, denoted $\alpha(G)$ or just $\alpha$. The independence sequence of $G$ is the list $s_{0}, s_{1}, s_{2}, \ldots, s_{\alpha}$ where $s_{i}$ is the number of independent sets of cardinality $i$, and the independence polynomial $S(G, z)$ is the generating function of the independence sequence, thus

$$
S(G, z)=\sum_{i=0}^{\alpha} s_{i} z^{i} .
$$


Note that the degree of $S(G, z)$ is $\alpha(G)$. Also, by convention, we set $s_{0}=1$.

Definition 1.1 (any-ordered). For a collection of graphs with the same independence number, we say that the independence sequence for the collection of graphs is any-ordered on the index set $\left\{i_{1}, \ldots, i_{\ell}\right\}$ if for any permutation $\pi$ of the set $\left\{i_{1}, \ldots, i_{\ell}\right\}$, there exists a graph in the collection with independence sequence $s_{0}, s_{1}, \ldots, s_{\alpha}$ such that

$$
s_{\pi\left(i_{1}\right)}<s_{\pi\left(i_{2}\right)}<\cdots<s_{\pi\left(i_{\ell}\right)} .
$$

The basic question that has motivated the work of this paper and others $([1,6])$ is: when is part of the independence sequence for a collection of graphs any-ordered?

In 1987 Alavi, Malde, Schwenk, and Erdős [1] showed that the independence sequence for all graphs with independence number $\alpha$ is any-ordered on the index set $\{1,2, \ldots, \alpha\}$ (this is the largest index set possible, since $s_{0}=1 \leq s_{i}$ for all $i \leq \alpha$ ). Theorem 2.2 provides a simple and short proof of this result using the power magnification operation.

Definition 1.2 (well-covered). A graph $G$ is well-covered if every maximal independent set has the same cardinality $\alpha(G)$. Equivalently, $G$ is well-covered if every maximal independent set is also a maximum independent set.

Some simple examples of well-covered graphs include complete graphs, the disjoint union of complete graphs, and cycles on 1, 2, 3, 4, 5, or 7 vertices (all other cycles are not well-covered).

It was conjectured in [2] that well-covered graphs have unimodal independence sequences. However, in [6], Michael and Traves produced counter-examples to the unimodality conjecture, and they posed the following new conjecture:

Conjecture 1.3 (The Roller-Coaster Conjecture). (i) For any well-covered graph with independence number $\alpha$, the terms of the independence sequence strictly increase from $s_{0}$ to $s_{\lceil\alpha / 2\rceil}$, and

(ii) the independence sequence for well-covered graphs with independence number $\alpha$ is any-ordered on $\{\lceil\alpha / 2\rceil, \ldots, \alpha\}$.

Michael and Traves [6] proved the first part of Conjecture 1.3 in general and the second part for $\alpha \leq 7$; however the rest remains open. Also one should note that while the unimodality conjecture is false in general, there do exist subclasses of well-covered graphs where the unimodality conjecture holds true, for example on certain kinds of trees - see [5] for details.

In Corollary 3.2, we will prove that for a given $\ell$, there exists sufficiently large $\alpha$ such that for well-covered graphs with independence number $\alpha$, the last $\ell$ terms of the independence sequence are any-ordered. Our main result is Theorem 3.1, in which we prove that as $\alpha$ gets large for well-covered graphs, at least the last (.1705) $\alpha$ terms in the independence sequence are any-ordered.

In Section 2 we will define the power magnification operation and show that it preserves well-coveredness (see Section 2.2), and we will then use power magnification to give a short and simple proof of Theorem 2.2, originally due to Alavi, Malde, Schwenk, and Erdős [1]. 
In Section 3 we state our main theorem (Theorem 3.1) and prove some general results relating to the Roller-Coaster Conjecture, including Proposition 3.6 which is a proof of the Roller-Coaster Conjecture for independence number $\alpha \leq 11$. Section 4 is dedicated to proving the main theorem (Theorem 3.1). Note that in Sections 2, 3, and 4 we make use of "formal" graphs; however, in Section 2.2 it is shown for any "formal" graph exhibiting a certain linear ordering of its independence sequence, there exists a bona fide graph with independence sequence exhibiting the same linear ordering. In Section 5, we develop two new well-coveredness-preserving operations, the partial join (a generalization of both the disjoint union and the join) and subgraph miniaturization. Finally, in Section 6 we give some possible directions for future research.

\section{Power Magnification}

Before defining the power magnification operation, we will discuss the disjoint union and the join, two simple well-coveredness preserving operations.

\subsection{Disjoint Union and the Join}

Let $G_{1}$ and $G_{2}$ be well-covered graphs. Then the disjoint union $G_{1} \amalg G_{2}$ is also wellcovered. Furthermore, we have the following formula for the independence number of the resulting graph:

$$
S\left(G_{1} \amalg G_{2}, z\right)=S\left(G_{1}, z\right) S\left(G_{2}, z\right) .
$$

For proof, see Michael and Traves [6].

Let $G_{1}$ and $G_{2}$ be graphs with the same independence number $\alpha$. Then the join of $G_{1}$ and $G_{2}$ (each vertex of $G_{1}$ is joined to each vertex in $G_{2}$ ), which we denote by $G_{1}+G_{2}$, is also well-covered. Furthermore,

$$
S\left(G_{1}+G_{2}, z\right)=S\left(G_{1}, z\right)+S\left(G_{2}, z\right)-1 .
$$

For proof, see Michael and Traves [6].

\subsubsection{Scaling independence polynomials by positive rational numbers}

For the purpose of determining possible linear orderings of the coefficients of a polynomial, it is only the relative sizes, not the absolute sizes, of the coefficients that matter. Thus, we will sometimes multiply an independence polynomial by a rational constant. The resulting scaled graph that has such an independence polynomial is denoted and defined as follows: $\frac{p}{q} G$ is to be interpreted as the join of $p$ copies of $G$ scaled down by actor of

$q$; hence, $S\left(\frac{p}{q} G, z\right)=\frac{p}{q} S(G, z)$. Note that at the end of this construction, we can always clear off denominators to get a bona fide graph and a bona fide independence polynomial, all while maintaining the linear ordering of the coefficients. 


\subsection{Definition of power magnification}

Let $G$ be a well-covered graph with independence number $\alpha$. Let

$$
H_{c}=\coprod_{i=1}^{n} K_{c}
$$

be the disjoint union of $n$ copies of $K_{c}$ for some large $c$. Also, define the graph $G$ power magnified by $n$ to be $H_{c} \amalg G$. Finally, we say that a sequence of polynomials converges to a polynomial $f$ if the coefficients of the sequence converge to the corresponding coefficients of $f$.

Proposition 2.1. Let $G_{c}$ denote the graph $G$ power magnified by $n$. Then $G_{c}$ is wellcovered and we have

$$
\lim _{c \rightarrow \infty} \frac{S\left(G_{c}, z\right)}{c^{n}}=z^{n} S(G, z) .
$$

Proof. Since both $G$ and $H_{c}$ are well-covered and disjoint unions of well-covered graphs are well-covered, $G_{c}$ is well-covered with independence number $\alpha+n$.

From Section 2.1, we know that

$$
S\left(H_{c} \amalg G, z\right)=S\left(H_{c}, z\right) S(G, z)=\left(\sum_{i=0}^{n}\left(\begin{array}{c}
n \\
i
\end{array}\right)(c z)^{i}\right) S(G, z) .
$$

Thus, Equation (1) is simply a consequence of the fact that $\frac{1}{c^{n}} \sum_{i=0}^{n}\left(\begin{array}{c}n \\ i\end{array}\right)(c z)^{i} \rightarrow z^{n}$ as $c \rightarrow \infty$.

We will use $z^{n} G$ to denote the limit graph of $G$ power magnified by $n$, which is defined to be the "formal" graph with independence polynomial $z^{n} S(G, z)=\lim _{c \rightarrow \infty} S\left(G_{c}, z\right)$. It is, of course, possible that there exists no bona fide graph with exactly this independence polynomial; however, we can find graphs that approximate the independence polynomial as accurately as we like. In particular, if the independence polynomial of the "formal" graph $z^{n} G$ is $s_{0}+s_{1} z+\cdots+s_{\alpha+n} z^{\alpha+n}$, then for each $\epsilon>0$ there exists sufficiently large $c$ such that the bona fide graph $G_{c}$ has independence polynomial $b_{0}+b_{1} z+\cdots+b_{\alpha+n} z^{\alpha+n}$ and

$$
\left|\frac{b_{i}}{c^{n}}-s_{i}\right|<\epsilon
$$

for all $i$. Thus, if the coefficients of $z^{n} G$ exhibit a particular linear ordering, say

$$
s_{\pi(0)}<s_{\pi(1)}<\cdots<s_{\pi(\alpha+n)}
$$

for some permutation $\pi$, then there exists sufficiently large $c$ such that $G_{c}$ exhibits the same linear ordering (note that scaling by $\frac{1}{c^{n}}$ does not change the linear ordering of the coefficients). In this way, $z^{n} G$ can be viewed simply as a computational place-holder for the scaled graph $\frac{1}{c^{n}} G_{c}$ with $c$ chosen sufficiently large. 


\subsection{An application of power magnification: independence se- quences of not-necessarily well-covered graphs}

The following theorem was proven by Alavi, Malde, Schwenk, and Erdős in [1]. We give a proof that constructs the same graph as that used in [1]; however, viewing the construction from the standpoint of power magnification results in a much shorter and cleaner proof.

Theorem 2.2 (Alavi, Malde, Schwenk, and Erdős). The independence sequence for (not necessarily well-covered) graphs of independence number $\alpha$ is any-ordered on the index set $\{1, \ldots, \alpha\}$.

Proof. Note that we may add independence polynomials of different degrees, corresponding to joining graphs of different independence number (this results in a non-well-covered graph).

Applying power magnification to $E_{0}$, the empty graph with no vertices or edges, yields a useful independence polynomial: $S\left(E_{0}, z\right)=1$, and so $S\left(z^{n} E_{0}, z\right)=z^{n}$.

Given $\pi$ a permutation of $\{1,2,3, \ldots, \alpha\}$, we may add polynomials $S\left(z^{n} E_{0}, z\right)$ (via the join operation) to get

$$
\begin{aligned}
P(z) & =1+1 S\left(z^{\pi(1)} E_{0}, z\right)+2 S\left(z^{\pi(2)} E_{0}, z\right)+\cdots+\alpha S\left(z^{\pi(\alpha)} E_{0}, z\right) \\
& =1+1 z^{\pi(1)}+2 z^{\pi(2)}+\cdots+\alpha z^{\pi(\alpha)} .
\end{aligned}
$$

Note that the graph with independence polynomial $P(z)$ (or a graph approximating $P(z)$ closely) has the property that $s_{\pi(i)}=i$ for all $i=1, \ldots, \alpha$, which clearly gives $s_{\pi(1)}<$ $s_{\pi(2)}<\cdots<s_{\pi(\alpha)}$.

\section{The Roller-Coaster Conjecture}

Our main theorem is a first step towards proving an asymptotic version of the RollerCoaster Conjecture.

Theorem 3.1 (Main Theorem). Define $k_{\alpha}$ to be the largest integer such that the last $k_{\alpha}$ terms of the independence sequence for well-covered graphs with independence number $\alpha$ are any-ordered. Then for all sufficiently large $\alpha$, we have

$$
(.1705) \alpha \leq k_{\alpha} \leq \frac{1}{2} \alpha .
$$

Theorem 3.1 of course gives us the following result:

Corollary 3.2. For any given $\ell$, there exists $\alpha$ large enough such that the last $\ell$ terms of the independence sequence are any-ordered for well-covered graphs with independence number $\alpha$. 
We will prove Theorem 3.1 in Section 4 by a construction that does slightly better than the given lower bound. The basic idea of the proof is to use power magnification along with the join and disjoint union to construct a graph that has the last (.1705) $\alpha$ terms very close to being equal. It is then possible to show that, by altering the proportions in which components of the graph are joined together, that the almost-equal terms in the independence sequence are in fact any-ordered. This is the same idea as used in [6] to prove the Roller-Coaster Conjecture for $\alpha \leq 7$ : in fact, for small $\alpha$ it is possible to construct graphs in which $s_{\lceil\alpha / 2\rceil}=\cdots=s_{\alpha}$.

The construction in Section 4 was tested by computer up to independence number $\alpha=500$, and it was found that roughly the last .2 $\alpha$ terms in the sequence were anyordered. Thus, the lower bound given in the Theorem 3.1 seems to be close to tight for the construction given in Section 4. Of course, it is possible that there exists another construction with a better lower bound.

The Roller-Coaster Conjecture implies that there exist graphs in which $s_{\lceil\alpha / 2\rceil}=\cdots=$ $s_{\alpha}$, and a natural open question is whether the converse is also true. That is, does the existence of a flat roller-coaster polynomial imply the second part of the Roller-Coaster Conjecture? We give a partial answer in the proposition below.

Theorem 3.3. Let $G$ be a well-covered graph with independence polynomial $S(G, z)=$ $p_{0}+p_{1} z+\cdots+p_{\alpha} z^{\alpha}$ and let $p_{i}=p_{j}$ for all $i, j \in J$, where $J$ is some index set. For a polynomial $f$, let $\left.f\right|_{J}$ be the polynomial consisting of all terms in $f$ with degree in $J$, so for example, $\left.S(G, z)\right|_{J}=\sum_{i \in J} p_{i} z^{i}$. If $G$ is the join of a well-covered graph $H$ to $|J|$ well-covered graphs $\left\{G_{i}\right\}$ where the polynomials $\left\{\left.S\left(G_{i}, z\right)\right|_{J}\right\}$ are linearly independent, then the independence sequence for well-covered graphs with independence number $\alpha$ is any-ordered on $J$.

Proof. Consider the $|J|$ linearly-independent vectors formed by listing the coefficients of the polynomials $\left\{\left.S\left(G_{i}, z\right)\right|_{J}\right\}$. Let $M$ be the $|J| \times|J|$-matrix having these vectors as columns. Linear independence implies that $M^{-1}$ exists, and we also know that $M^{-1}$ is continuous.

Let $\mathbf{x}=\left(x_{1}, \ldots, x_{|J|}\right)^{\mathrm{t}}$, where $x_{i}$ is the (positive) number of copies of $G_{i}$ that were joined to make $G$. Thus, $M(\mathbf{x})$ lists the terms of the independence sequence of $G \backslash H$ with indices in $J$ (note $G \backslash H$ is subgraph of $G$ composed of joins of the graphs $\left\{G_{i}\right\}$ ). Letting $\mathbf{h}$ be the vector defined by $\left.S(H, z)\right|_{J}$, we have that $M(\mathbf{x})+\mathbf{h}$ lists of the terms of the independence sequence of $G$ with indices in $J$ and thus is constant. Since $x_{i}>0$ for all $i$ and $M^{-1}$ is continuous, we know that there exists $\delta>0$ such that for every vector $\mathbf{y}$ satisfying $|\mathbf{y}-M(\mathbf{x})|<\delta$, we have that every coordinate of $M^{-1}(\mathbf{y})$ is positive. Hence $M^{-1}(\mathbf{y})$ lists the (positive) number of copies of each $G_{i}$ we would need to join to $H$ in order to get a graph with the property that $\mathbf{y}+\mathbf{h}$ lists the terms of the graph's independence sequence with indices in $J$. Note that we can make $M^{-1}(\mathbf{y})$ rational by choosing $\mathbf{y}$ to be rational, and by Section 2.1.1 we can easily deal with fractional copies. Since $M(\mathbf{x})+\mathbf{h}$ is constant, we can choose a vector $\mathbf{y}$ so that $|\mathbf{y}-M(\mathbf{x})|<\delta$ and $\mathbf{y}+\mathbf{h}$ has any desired linear ordering, and hence we are done. 
We next prove some results that might be useful in trying to develop an inductive proof of the Roller-Coaster Conjecture.

Theorem 3.4. If the last $n$ terms of the independence sequence for well-covered graphs are any-ordered for a given $\alpha_{o}$, then the last $n$ terms of the independence sequence are any-ordered for all $\alpha>\alpha_{o}$.

Proof. Say $G$ is a graph that exhibits a given permutation $\pi$ of the last $n$ indices in its independence sequence. Then $z G$ (the limit graph of $G$ power magnified by 1 ) exhibits the permutation $\pi$ on its last $n$ indices as well. By induction, this completes the proof.

Corollary 3.5. If the Roller-Coaster Conjecture is true for a given even $\alpha$, then the Roller-Coaster Conjecture is also true for $\alpha+1$.

Proof. Apply Theorem 3.4 and use the fact that $\left\lceil\frac{\alpha+1}{2}\right\rceil=\left\lceil\frac{\alpha}{2}\right\rceil+1$.

Proposition 3.6. The Roller-Coaster Conjecture (Conjecture 1.3) is true for $\alpha \leq 11$.

Proof. For $\alpha=1, \ldots, 7$, see Michael and Traves [6].

Following the method of Michael and Traves, we will construct flat roller-coaster polynomials meeting the conditions of Theorem 3.3 to prove the remaining cases. Notice that by Corollary 3.5, it is sufficient to construct flat roller-coaster polynomials for $\alpha=8$ and $\alpha=10$. We add (using joins) and multiply (using disjoint unions) polynomials of the form $S\left(z^{n} E_{0}, z\right)=z^{n}$ and of the form $S\left(\coprod_{1}^{\alpha-n} K_{1}, z\right)=(1+z)^{\alpha-n}$ to get

$$
\begin{aligned}
3(1+z)^{8}+14 z^{4}(1+z)^{4}+56 z^{6}(1+z)^{2}+32 z^{7}(1+z)+119 z^{8} \\
=3+24 z+84 z^{2}+168 z^{3}+224 z^{4}+224 z^{5}+224 z^{6}+224 z^{7}+224 z^{8}
\end{aligned}
$$

and

$$
\begin{gathered}
14(1+z)^{10}+9 z(1+z)^{9}+42 z^{5}(1+z)^{5}+420 z^{6}(1+z)^{4}+ \\
+168 z^{7}(1+z)^{3}+306 z^{8}(1+z)^{2}+1477 z^{9}(1+z)+2268 z^{10} \\
=14+149 z+711 z^{2}+2004 z^{3}+3696 z^{4}+4704 z^{5}+ \\
+4704 z^{6}+4704 z^{7}+4704 z^{8}+4704 z^{9}+4704 z^{10}
\end{gathered}
$$

which completes the proof. Note that similar constructions with the polynomials $z^{n}(1+$ $z)^{\alpha-n}$ can be used to prove the Roller-Coaster Conjecture for $\alpha=2,4$, and 6 .

One might hope to find a result similar to Corollary 3.5, showing, for example, that the Roller-Coaster Conjecture being true for a given odd $\alpha$ implies that it is true for the next even number. Of course, this would imply that the conjecture were is true for all $\alpha$, but unfortunately, such a result appears to be quite elusive.

\section{Proof of the main theorem (Theorem 3.1)}

The general idea of the proof is to use power magnification to create independence polynomials of the form $z^{i}(1+z)^{\alpha-i}$ and to add these together in such a way that the resulting polynomial has all coefficients of degree (.1705) $\alpha$ or higher almost equal. We will then show that we can alter the construction slightly so that coefficients of degree (.1705) $\alpha$ or higher are exactly equal, which then proves Theorem 3.1 by way of Theorem 3.3. 


\subsection{The almost flat roller-coaster polynomial construction}

Define the graphs

$$
H_{\alpha, i}=z^{i}\left(\coprod_{1}^{\alpha-i} K_{1}\right)
$$

for $i=0,1,2, \ldots, \alpha$. Note that all the $H_{\alpha, i}$ have independence number $\alpha$, which means that well-coveredness is preserved when taking joins of the $H_{\alpha, i}$.

We define the almost-flat roller-coaster graph to be

$$
F_{\alpha}=\frac{1}{2^{\alpha}} H_{\alpha, 0}+\sum_{i=1}^{\alpha} \frac{1}{2^{\alpha-i+1}} H_{\alpha, i}
$$

It is clear that $F_{\alpha}$ is well-covered with independence number $\alpha$. The independence polynomial of $F_{\alpha}$, which we call the almost-flat roller-coaster polynomial, is

$$
S\left(F_{\alpha}, z\right)=\left(\frac{1+z}{2}\right)^{\alpha}+\sum_{i=1}^{\alpha} \frac{z^{i}(1+z)^{\alpha-i}}{2^{\alpha-i+1}} .
$$

Notice that we have the following relationship:

$$
S\left(F_{\alpha+1}, z\right)=S\left(F_{\alpha}, z\right) \frac{1+z}{2}+\frac{z^{\alpha+1}}{2} .
$$

Thus, we can interpret the construction of the polynomial $S\left(F_{\alpha}, z\right)$ as an iterative process in which each step consists of averaging all adjacent coefficients (including the constant and highest-degree coefficients with zero) and then correcting the new highest degree coefficient to be one.

Notation: For a polynomial $p(z)=p_{0}+p_{1} z+\cdots+p_{n} z^{n}$, we define the vector $[p(z)]=\left(p_{n}, p_{n-1}, \ldots, p_{1}, p_{0}\right)$. Notice that the coefficients are listed from highest to lowest degree. In Table 4.1 we list $\left[S\left(F_{\alpha}, z\right)\right]$ for a few small values of $\alpha$.

From the definition of the graphs $H_{\alpha, i}$ (Equation $\left.(2)\right)$, we get that $\left[S\left(H_{\alpha, \alpha-i+1}, z\right)\right]$ consists of the $i$ th row of Pascal's Triangle followed by $\alpha-i+1$ zeroes. For example, $\left[S\left(H_{\alpha, \alpha-2}, z\right)\right]=(1,2,1,0,0,0, \ldots, 0)$ and $\left[S\left(H_{\alpha, \alpha-3}, z\right)\right]=(1,3,3,1,0,0, \ldots, 0)$. Thus, the vectors $\left\{\left[S\left(H_{\alpha, i}, z\right)\right]\right\}_{i=0}^{\alpha}$ are linearly independent.

We will use $\left\{\left[S\left(H_{\alpha, i}, z\right)\right]^{\mathrm{t}}\right\}$ as columns to define the invertible $(\alpha+1) \times(\alpha+1)$ matrix

$$
M=\left(\begin{array}{cccccccc}
1 & 1 & 1 & 1 & 1 & 1 & 1 & \cdots \\
0 & 1 & 2 & 3 & 4 & 5 & 6 & \ldots \\
0 & 0 & 1 & 3 & 6 & 10 & 15 & \ldots \\
0 & 0 & 0 & 1 & 4 & 10 & 20 & \cdots \\
0 & 0 & 0 & 0 & 1 & 5 & 15 & \cdots \\
0 & 0 & 0 & 0 & 0 & 1 & 6 & \ldots \\
0 & 0 & 0 & 0 & 0 & 0 & 1 & \cdots \\
\vdots & \vdots & \vdots & \vdots & \vdots & \vdots & \vdots & \ddots
\end{array}\right) .
$$




\begin{tabular}{|c|c|c|}
\hline$\alpha$ & {$\left[S\left(F_{\alpha}, z\right)\right]$} & \\
\hline 0 & $(1)$ & $=1 \cdot(1)$ \\
\hline 1 & $\left(1, \frac{1}{2}\right)$ & $=\frac{1}{2} \cdot(2,1)$ \\
\hline 2 & $\left(1, \frac{3}{4}, \frac{1}{4}\right)$ & $=\frac{1}{4} \cdot(4,3,1)$ \\
\hline 3 & $\left(1, \frac{7}{8}, \frac{4}{8}, \frac{1}{8}\right)$ & $=\frac{1}{8} \cdot(8,7,4,1)$ \\
\hline 4 & $\left(1, \frac{15}{16}, \frac{11}{16}, \frac{5}{16}, \frac{1}{16}\right)$ & $=\frac{1}{16} \cdot(16,15,11,5,1)$ \\
\hline 5 & $\left(1, \frac{31}{32}, \frac{26}{32}, \frac{16}{32}, \frac{6}{32}, \frac{1}{32}\right)$ & $=\frac{1}{32} \cdot(32,31,26,16,6,1)$ \\
\hline 6 & $\left(1, \frac{63}{64}, \frac{57}{64}, \frac{42}{64}, \frac{22}{64}, \frac{7}{64}, \frac{1}{64}\right)$ & $=\frac{1}{64} \cdot(64,63,57,42,22,7,1)$ \\
\hline & & \\
\hline
\end{tabular}

Table 1: Here we list the coefficients for the almost flat roller-coaster polynomial $S\left(F_{\alpha}, z\right)$ for small $\alpha$. Note that by convention, the vector $\left[S\left(F_{\alpha}, z\right)\right]$ lists the coefficients in order from highest degree to lowest degree.

By expanding Equation (3), we see that the vector $\mathbf{s} \in \mathbb{Q}^{\alpha+1}$ such that $M(\mathbf{s})=\mathbf{f}=$ $\left[F_{\alpha}(z)\right]$ is

$$
\mathbf{s}=\left(2^{-1}, 2^{-2}, 2^{-3}, \ldots, 2^{-\alpha+1}, 2^{-\alpha}, 2^{-\alpha}\right) .
$$

\subsection{Approximations that prove Theorem 3.1}

Proving the upper bound in Theorem 3.1 is simply the first part of the Roller-Coaster Conjecture, which was proven in [6].

To prove the lower bound we will show that $F_{\alpha}$, the almost-flat roller-coaster graph, can be perturbed slightly to create a graph with an independence polynomial that is completely flat on slightly more than the last $(.1705) \alpha$ coefficients. Theorem 3.3 then proves any-orderedness.

We need a way to calculate the $n$th highest degree coefficient of $S\left(F_{\alpha}, z\right)$, where $F_{\alpha}$ is the almost-flat graph from Section 4.1. Recall that in the iterative interpretation of constructing the polynomial $S\left(F_{\alpha}, z\right)$, we successively average adjacent coefficients. Thus, Table 2 shows the local behavior of the coefficients of $\left[S\left(F_{\alpha}, z\right)\right]$ (which lists the coefficients in order from highest degree to lowest degree) starting from independence number $\alpha_{o}$. Notice that the coefficients of the $k_{i}$ in each numerator in Table 2 form a row of Pascal's Triangle.

For a given $\alpha$, let $w_{n}$ denote the $n$th term in the sequence $\left[S\left(F_{\alpha}, z\right)\right]$. Assume in Table 2 that $\alpha_{o}=0$ and also set $k_{i}=1$ for $i \leq 1$, and $k_{i}=0$ for $i>1$. Then the first pictured column in Table 2 (headed by $k_{1}$ ) is $w_{1}$, the second column (headed by $k_{2}$ ) is $w_{2}$, and so on. One can see that in general,

$$
\begin{aligned}
w_{n} & =\frac{1}{2^{\alpha}}\left(k_{-\alpha+n}+\left(\begin{array}{l}
\alpha \\
1
\end{array}\right) k_{-\alpha+n+1}+\cdots+\left(\begin{array}{c}
\alpha \\
\alpha-n+1
\end{array}\right) k_{1}+\left(\begin{array}{c}
\alpha \\
\alpha-n+2
\end{array}\right) k_{2}+\cdots+k_{n}\right) \\
& =\frac{1}{2^{\alpha}}\left(1+\left(\begin{array}{l}
\alpha \\
1
\end{array}\right)+\cdots+\left(\begin{array}{c}
\alpha \\
\alpha-n+1
\end{array}\right)\right) .
\end{aligned}
$$


Table 2: Below we give the local behavior of the almost flat roller-coaster algorithm on $\left[S\left(F_{\alpha}, z\right)\right]$ for $\alpha=\alpha_{o}$ to $\alpha_{o}+4$. The $k_{i}$ are the coordinates of $\left[S\left(F_{\alpha_{o}}, z\right)\right]$.

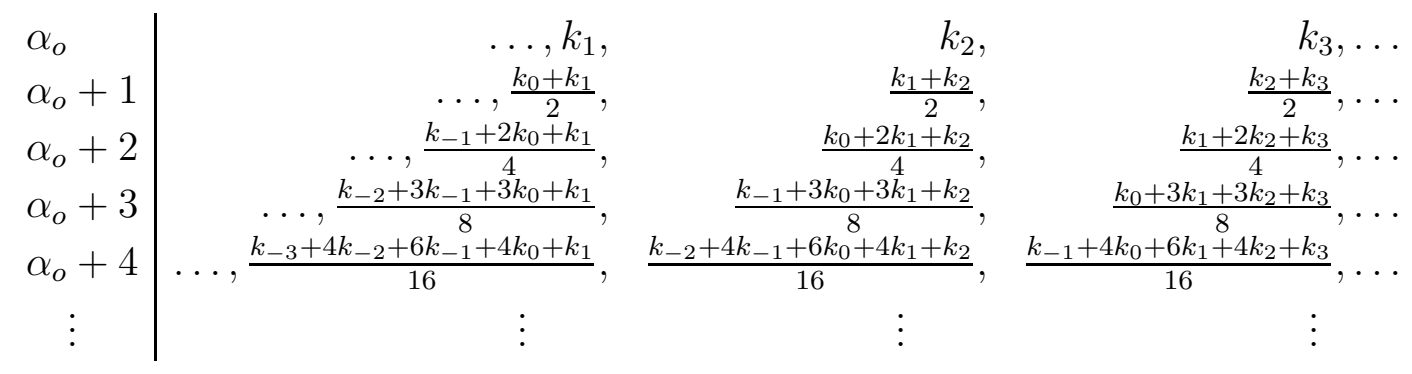

Note that $\left[S\left(F_{\alpha}, z\right)\right]=\mathbf{f}=\left(w_{1}, w_{2}, \ldots, w_{\alpha+1}\right)$. Let the vector $\mathbf{y}_{\mathbf{n}}=\left(z_{1}, z_{2}, \ldots, z_{\alpha+1}\right)$ be defined by

$$
z_{i}=\left\{\begin{array}{l}
1 \text { for } 1 \leq i \leq n \\
w_{i} \text { for } n<i \leq \alpha+1
\end{array}\right.
$$

If we satisfy the inequality

$$
\left\|M^{-1}\left(\mathbf{y}_{\mathbf{n}}\right)-M^{-1}(\mathbf{f})\right\| \leq \frac{1}{2^{n}}
$$

then we know, since $M^{-1}(\mathbf{f})=\mathbf{s}=\left(2^{-1}, 2^{-2}, \ldots, 2^{-\alpha}, 2^{-\alpha}\right)$ and $\mathbf{y}_{\mathbf{n}}$ and $\mathbf{f}$ differ only on the first $n$ coordinates, that all the coordinates of $\mathbf{x}=M^{-1}\left(\mathbf{y}_{\mathbf{n}}\right)$ are positive . This means in turn that $\mathbf{y}_{\mathbf{n}}$ is a linear combination of the vectors $\left\{\left[S\left(H_{\alpha, i}, z\right)\right]\right\}$ with positive coefficients ( since $M(\mathbf{x})=\mathbf{y}_{\mathbf{n}}$ ), which gives us a construction for the completely flat polynomial represented by $\mathbf{y}_{\mathbf{n}}$. Thus, proving that Inequality (4) holds for sufficiently large $\alpha$ completes the proof.

There is a simple way to bound $\left\|M^{-1}\left(\mathbf{y}_{\mathbf{n}}\right)-M^{-1}(\mathbf{f})\right\|$, namely:

$$
\begin{aligned}
\left\|M^{-1}\left(\mathbf{y}_{\mathbf{n}}\right)-M^{-1}(\mathbf{f})\right\| & =\left\|M^{-1}\left(\mathbf{y}_{\mathbf{n}}-\mathbf{f}\right)\right\| \\
& =\left\|M^{-1}\left(\left(1-w_{1}, 1-w_{2}, \ldots, 1-w_{n}, 0,0,0, \ldots\right)\right)\right\| \\
& <\max _{1 \leq i \leq n}\left(\left\{1-w_{i}\right\}\right) n^{2} \max \left(\left\{\left|m_{i j}\right|: m_{i j} \in M^{-1}, 1 \leq i, j \leq n\right\}\right) \\
& <\max _{1 \leq i \leq n}\left(\left\{1-w_{i}\right\}\right) n^{2} 2^{n} \\
& <\left(1-w_{n}\right) n^{2} 2^{n} .
\end{aligned}
$$

(Note that the $w_{i}$ are strictly increasing as $i$ increases from 1 to $\alpha / 2$.) The $n^{2}$ comes from the fact that there are $n^{2}$ entries in the upper left $n \times n$ block of $M^{-1}$, each of which is less than or equal to the maximum entry. The $2^{n}$ in the last inequality comes from directly 
computing the inverse of $M$ :

$$
M^{-1}=\left(\begin{array}{cccccccl}
1 & -1 & 1 & -1 & 1 & -1 & 1 & \ldots \\
0 & 1 & -2 & 3 & -4 & 5 & -6 & \ldots \\
0 & 0 & 1 & -3 & 6 & -10 & 15 & \cdots \\
0 & 0 & 0 & 1 & -4 & 10 & -20 & \cdots \\
0 & 0 & 0 & 0 & 1 & -5 & 15 & \cdots \\
0 & 0 & 0 & 0 & 0 & 1 & -6 & \cdots \\
0 & 0 & 0 & 0 & 0 & 0 & 1 & \cdots \\
\vdots & \vdots & \vdots & \vdots & \vdots & \vdots & \ddots &
\end{array}\right)
$$

and then noting that the largest entry in the upper left $n \times n$ block of $M^{-1}$ is $\left(\begin{array}{c}n \\ \lceil n / 2\rceil\end{array}\right)<2^{n}$ (recall that the sum of the entries in the $n$th row of Pascal's Triangle is $2^{n}$ ).

Combining the above with Inequality (4), Theorem 3.1 is proven if we show that for sufficiently large $\alpha$,

$$
\left(1-w_{n}\right) n^{2} 2^{n}<\frac{1}{2^{n}} .
$$

Stirling's formula states (see [4], page 54) that

$$
\sqrt{2 \pi} m^{m+\frac{1}{2}} \exp \left(-m+\frac{1}{12 m+1}\right)<m !<\sqrt{2 \pi} m^{m+\frac{1}{2}} \exp \left(-m+\frac{1}{12 m}\right),
$$

where $\exp (x)=e^{x}$.

We will use Stirling's formula to approximate $\left(\begin{array}{c}\alpha \\ i\end{array}\right)$ for various $i$, and thereby find an upper bound for $1-w_{n}$.

$$
\begin{aligned}
\left(\begin{array}{c}
\alpha \\
m
\end{array}\right) & =\frac{\alpha !}{m !(\alpha-m) !} \\
& <\frac{\sqrt{2 \pi} \alpha^{\alpha+\frac{1}{2}} \exp \left(-\alpha+\frac{1}{12 \alpha}\right)}{\sqrt{2 \pi} m^{m+\frac{1}{2}} \exp \left(-m+\frac{1}{12 m+1}\right) \sqrt{2 \pi}(\alpha-m)^{\alpha-m+\frac{1}{2}} \exp \left(-\alpha+m+\frac{1}{12(\alpha-m)+1}\right)} \\
& =\frac{\alpha^{\alpha+\frac{1}{2}} \exp \left(\frac{1}{12 \alpha}-\frac{1}{12 m+1}-\frac{1}{12(\alpha-m)+1}\right)}{\sqrt{2 \pi} m^{m+\frac{1}{2}}(\alpha-m)^{\alpha-m+\frac{1}{2}}}
\end{aligned}
$$

Thus we have

$$
1-w_{n}=2^{-\alpha} \sum_{i=\alpha-n+1}^{\alpha}\left(\begin{array}{c}
\alpha \\
i
\end{array}\right)<2^{-\alpha} n\left(\begin{array}{c}
\alpha \\
n-1
\end{array}\right) .
$$

We want to find the largest possible $n$, and so we set $n=\alpha-\beta \alpha$ where $\frac{1}{2}<\beta<1$. 
Using the above computation we have the following bound:

$$
\begin{aligned}
\left(\begin{array}{c}
\alpha \\
n-1
\end{array}\right) & \leq\left(\begin{array}{l}
\alpha \\
n
\end{array}\right) \\
& <\frac{\alpha^{\alpha+\frac{1}{2}} \exp \left(\frac{1}{12 \alpha}-\frac{1}{12(\alpha-\beta \alpha)+1}-\frac{1}{12 \beta \alpha+1}\right)}{\sqrt{2 \pi}(\alpha-\beta \alpha)^{\alpha-\beta \alpha+\frac{1}{2}}(\beta \alpha)^{\beta \alpha+\frac{1}{2}}} \\
& =\frac{\alpha^{-\frac{1}{2}} c_{e}(\alpha, \beta)}{\sqrt{2 \pi} c_{d}(\alpha, \beta)}
\end{aligned}
$$

where

$$
c_{e}(\alpha, \beta)=\exp \left(\frac{1}{12 \alpha}-\frac{1}{12(\alpha-\beta \alpha)+1}-\frac{1}{12 \beta \alpha+1}\right)
$$

and

$$
c_{d}(\alpha, \beta)=(1-\beta)^{\alpha-\beta \alpha+\frac{1}{2}}(\beta)^{\beta \alpha+\frac{1}{2}} .
$$

Thus, using Inequality (6), we have

$$
1-w_{n}<\frac{2^{-\alpha} n \alpha^{-\frac{1}{2}} c_{e}(\alpha, \beta)}{\sqrt{2 \pi} c_{d}(\alpha, \beta)} .
$$

Lemma 4.1. For any given $\alpha$, if $\frac{1}{2}<\beta<1$, then $c_{e}(\alpha, \beta)<1$.

Proof. Since $e^{x}$ is an increasing function, it is sufficient to show that

$$
f(\beta)=\frac{1}{12 \alpha}-\frac{1}{12(\alpha-\beta \alpha)+1}-\frac{1}{12 \beta \alpha+1}<0
$$

whenever $\frac{1}{2}<\beta<1$.

Taking a derivative of $f(\beta)$, we can show that there are three critical points, one of which is $\frac{1}{2}$, one of which is less than $\frac{1}{2}$ and one of which is greater than 1 . Since $f^{\prime}(1)<0$ this shows that $f(\beta)$ is decreasing for $\frac{1}{2}<\beta \leq 1$.

Plugging in $\beta=\frac{1}{2}$ thus gives us an upper bound for $f(\beta)$, namely

$$
f(\beta) \leq \frac{1}{12 \alpha}-\frac{2}{6 \alpha+1}<0 .
$$

Recall that it suffices to prove Theorem 3.1 to show that Inequality (5), which is equivalent to $\left(1-w_{n}\right) n^{2} 2^{2 n}<1$, holds for for sufficiently large $\alpha$. Using the bounds from above and the fact that $n=\alpha-\beta \alpha$ (by definition of $\beta$ ), we have that

$$
\begin{aligned}
\left(1-w_{n}\right) n^{2} 2^{2 n} & <2^{-\alpha} n^{3} 2^{2 n} \frac{\alpha^{-1 / 2} c_{e}(\alpha, \beta)}{\sqrt{2 \pi} c_{d}(\alpha, \beta)} & & \text { (by Inequality (7)) } \\
& <\frac{2^{\alpha-2 \beta \alpha} \alpha^{\frac{-5}{2}}}{\sqrt{2 \pi} c_{d}(\alpha, \beta)} & & \text { (by definition of } \beta \text { and Lemma 4.1). }
\end{aligned}
$$


Substituting in the definition of $c_{d}$ and re-arranging the terms, it is sufficient to show that for sufficiently large $\alpha$, we have

$$
\left(\frac{2^{1-2 \beta}}{(1-\beta)^{(1-\beta)} \beta^{\beta}}\right)^{\alpha}<\alpha^{\frac{5}{2}} \sqrt{2 \pi} \beta^{\frac{1}{2}}(1-\beta)^{\frac{1}{2}} .
$$

Thus, we need to choose the fixed constant $\beta$ such that

$$
\frac{2^{1-2 \beta}}{(1-\beta)^{(1-\beta)} \beta^{\beta}}<1,
$$

which is equivalent to

$$
0<(2 \beta-1) \ln 2+(1-\beta) \ln (1-\beta)+\beta \ln (\beta) .
$$

We note that Inequality ( 8 ) is satisfied by $\beta=.82946434$. This proves that there exists sufficiently large $\alpha$ such that the independence sequence for well-covered graphs with independence number $\alpha$ is any-ordered on the last .17053566 $\alpha$ terms of the independence sequence.

\section{Well-coveredness preserving operations}

In this section we define two new well-coveredness preserving operations, the partial join and subgraph miniaturization, both of which have easily computable independence polynomials.

It is well-known that a graph $G$ is well-covered of independence number $\alpha$ if and only if for each vertex $v$, the induced $G-N[v]$ is well-covered of independence number $\alpha-1$, where $N[v]$ denotes $v$ and all of its neighbors. Thus, a well-covered graph contains many well-covered induced subgraphs. However, it is not true that every induced subgraph of a well-covered graph is well-covered. In fact, given an arbitrary graph $G$, it is possible to construct a well-covered graph that has $G$ as an induced subgraph.

Proposition 5.1. Given an arbitrary graph $G$ with $n$ vertices and a complete graph $K_{m}$ where $m \geq n$, the Cartesian product $G \times K_{m}$ is well-covered with independence number $n$.

Proof. In Figure 1 we have arranged the vertices so that each of the $m$ columns is a copy of $G$ and each of the $n$ rows is a copy of $K_{m}$. Let $I$ be a maximal independent set; no two vertices in $I$ can be in the same row because each row is a complete subgraph, hence $|I| \leq n$.

If $|I|<n$, then there is a row with no vertices in $I$. Because there are at least as many columns as rows ( $m \geq n$ by assumption), there is also a column with no vertices in $I$. The vertex at the intersection of the row and the column with no vertices in $I$ is thus not adjacent to any vertices in $I$; hence $I$ is not maximal. This shows that every maximal independent set $I$ has cardinality $n$, which proves that $G \times K_{m}$ is well-covered.

In general, the Cartesian product does not result in graphs whose independence polynomials may be easily calculated. In the remainder of this section, we will develop a few graph operations that lead to easily calculated independence polynomials. 


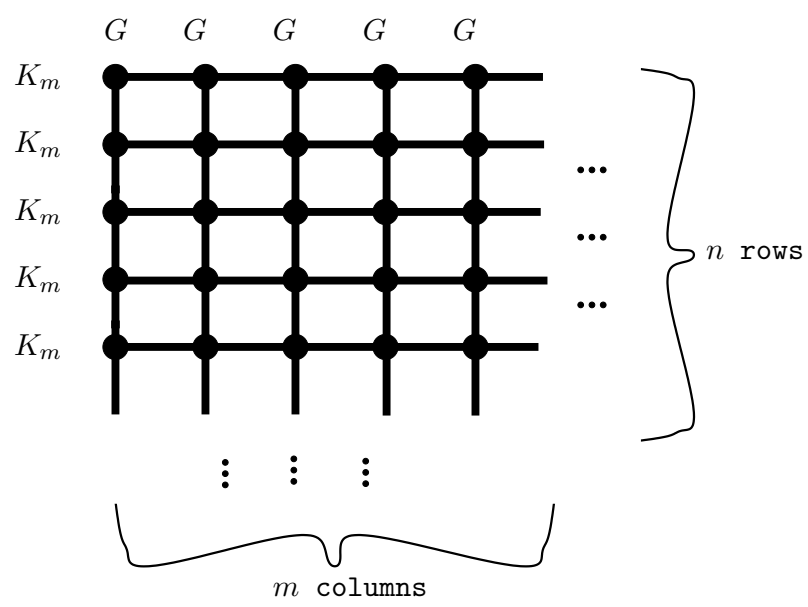

Figure 1: In this depiction of the Cartesian product $G \times K_{m}$, we have compressed the subgraphs so that all the vertices in each copy of $K_{m}$ and of $G$ are colinear, with the thick lines denoting the various edges that go between the vertices.

\subsection{Partial Join}

The partial join is a generalization of both the disjoint union and of the join as a single operation. We define the partial join algorithmically as follows.

1. Let $G_{1}$ and $G_{2}$ be well-covered with independence numbers $\alpha_{1}$ and $\alpha_{2}$, respectively.

2. Let $H_{1} \subset G_{2}$ and $H_{2} \subset G_{2}$ be well-covered induced subgraphs with independence numbers $\beta_{1}$ and $\beta_{2}$, respectively, such that $\alpha_{1}+\beta_{2}=\alpha_{2}+\beta_{1}$.

3. Join the vertices in $V\left(G_{1}\right) \backslash V\left(H_{1}\right)$ to those in $V\left(G_{2}\right) \backslash V\left(H_{2}\right)$ (while keeping all the edges originally in $G_{1}$ and $G_{2}$ ), and call the resulting graph, which we denote $G_{1}+{ }_{\left(H_{1}, H_{2}\right)} G_{2}$, the partial join of $G_{1}$ and $G_{2}$ avoiding $H_{1}$ and $H_{2}$.

Proposition 5.2. The partial join of $G_{1}$ and $G_{2}$ avoiding $H_{1}$ and $H_{2}$ is well-covered and we have

$$
S\left(G_{1}+{ }_{\left(H_{1}, H_{2}\right)} G_{2}, z\right)=S\left(G_{1}, z\right) S\left(H_{2}, z\right)+S\left(G_{2}, z\right) S\left(H_{1}, z\right)-S\left(H_{2}, z\right) S\left(H_{1}, z\right) .
$$

Proof. First we show that $G_{1}+{ }_{\left(H_{1}, H_{2}\right)} G_{2}$ is well-covered with independence number $\alpha_{1}+\beta_{2}$. Let $I$ be a maximal independent set in $G_{1}+{ }_{\left(H_{1}, H_{2}\right)} G_{2}$. Due to the joins of subgraphs, we know that $I$ is contained in either $G_{1} \amalg H_{2}$ or $G_{2} \amalg H_{1}$. In the first case $|I|=\alpha_{1}+\beta_{2}$ and in the second case $|I|=\alpha_{2}+\beta_{1}$ (note that both disjoint unions are well-covered graphs). Since $\alpha_{1}+\beta_{2}=\alpha_{2}+\beta_{1}$ by assumption, this proves that $G_{1}+_{\left(H_{1}, H_{2}\right)} G_{2}$ is well-covered.

Derivation of Equation (9): We may count independent sets in $G_{1}+_{\left(H_{1}, H_{2}\right)} G_{2}$ by considering two cases: any independent set is contained in either $G_{1} \amalg H_{2}$, or contained in $G_{2} \amalg H_{1}$. These two cases contribute $S\left(G_{1}, z\right) S\left(H_{2}, z\right)$ and $S\left(G_{2}, z\right) S\left(H_{1}, z\right)$, respectively, to the independence polynomial $S\left(G_{1}+{ }_{\left(H_{1}, H_{2}\right)} G_{2}, z\right)$. However, independent sets in $H_{1} \amalg H_{2}$ have been counted twice, and thus we must correct by subtracting $S\left(H_{1}, z\right) S\left(H_{2}, z\right)$. 
In addition to the disjoint union and the join, there are some other interesting special cases of the partial join. One interesting case is when the avoided subgraph of $G_{2}$ is $H_{2}=E_{0}$, the graph with no edges or vertices, which results in the formula:

$$
S\left(G_{1}+_{\left(H_{1}, E_{0}\right)} G_{2}, z\right)=S\left(G_{1}, z\right)+\left(S\left(G_{2}, z\right)-1\right) S\left(H_{1}, z\right) .
$$

Here one can think of the contribution of $H_{1}$ to the independence polynomial of $G_{1}$ being "magnified" by the independence polynomial of $G_{2}$. Another special case of the partial join is the well-known operation of vertex expansion (see [2]). One can think of vertex expansion as replacing a given vertex $v$ with a complete graph $K_{b}$ and then joining $K_{b}$ to all neighbors of $v$. This operation is the same as taking the partial join of $G$ and $K_{b-1}$ avoiding the induced subgraphs $V(G) \backslash N[v] \subset V(G)$ and $\emptyset \subset V\left(K_{b-1}\right)$.

\subsection{Subgraph Miniaturization}

The operation of subgraph miniaturization gives a way to subtract the independence polynomial of a subgraph from the independence polynomial of the entire graph. We define subgraph miniaturization algorithmically below.

1. Let $G$ be a well-covered graph with independence number $\alpha$, and let $H$ be any induced subgraph of $G$ ( $H$ need not be well-covered). Let $G \backslash H$ denote the induced subgraph of $G$ on $V(G) \backslash V(H)$.

2. Join each of $b-1$ copies of $G \backslash H$ with each other and with the original induced subgraph $G \backslash H$ of $G$.

3. Attach each copy of $G \backslash H$ to the lone copy of $H$ exactly as the original $G \backslash H$ was attached to $H$. Stated precisely, we label the vertices in $G \backslash H$ with $j=$ $1,2, \ldots,|G \backslash H|$, and we label all copies of $G \backslash H$ with $i=0,1, \ldots,(b-1)$ where the original $G \backslash H$ is labeled 0 . Referring to a vertex $j$ in copy $i$ of $G \backslash H$ by the ordered pair $(i, j)$, this step may be stated as: connect $(i, j)$ to vertex $v$ in $H$ if and only if $v$ is adjacent to $(0, j)$. Call the resulting graph $G$ with subgraph $H$ miniaturized, and denote it $G_{H}$.

Proposition 5.3. The graph $G$ with subgraph $H$ miniaturized is well-covered and we have

$$
S\left(G_{H}, z\right)=b S(G, z)-(b-1) S(H, z) .
$$

Proof. Let $I$ be a maximal independent set in $G_{H}$. If $I$ is contained in $H$, then $I$ must be a maximal independent set of the original graph $G$ (because $G$ is an induced subgraph of $G_{H}$ ) and so $I$ has cardinality $\alpha$. If $I$ contains any vertices not in $H$, then $I$ is contained in some single copy of $(G-H) \cup H$. By Step 3 above, $(G-H) \cup H$ is isomorphic to $G$; and hence $I$ again has cardinality $\alpha$.

Derivation of Equation (10): An independence set may be contained in at most one copy of $G \backslash H$, and since we may choose among $b$ copies, we multiply $S(G, z)$ by $b$. However, we do not have a choice of copies for independent sets contained in $H$, and so such independent sets should be counted only once, not $b$ times. Thus we correct by subtracting $b-1$ times $S(H, z)$. 


\section{$6 \quad$ Further questions}

Studying the possible linear orderings of independence sequences can be regarded as a step towards solving the more general problem:

Question 6.1. What are necessary and sufficient conditions for a sequence to be an independence sequence of some well-covered graph?

In this paper we attempted to characterize the constraints on independence sequences of well-covered graphs by studying the possible linear orderings of the terms. Another reasonable approach to take would be to study the possible relative sizes of terms in the independence sequence. One should notice that the almost flat polynomial construction forced all of the higher degree coefficients to have relative sizes that differed by only some small factor, even when we perturbed the polynomial to get different orderings on the last $n$ terms. It would be an interesting problem to find bounds on the relative sizes of terms of the independence sequence, especially if one were to keep the order of some or all of the terms fixed.

Some work has already been done on this approach; for instance, Michael and Traves proved in [6] that for well-covered graphs, the sharpest peak one could achieve at the $\lceil\alpha / 2\rceil$ th term of the independence sequence comes from the binomial expansion $(1+z)^{\alpha}$. Interestingly, no such constraints apply to the non-well-covered case. By modifying the proof of Theorem 2.2 slightly, it is easy to show that the independence sequence of a not necessarily well-covered graph is completely unconstrained with respect to relative size, as well as with respect to linear order.

There is also, of course, the second part of the Roller Coaster Conjecture (Conjecture 1.3) that remains to be proven for $\alpha>11$. One indication that the conjecture may be true, at least for sufficiently large $\alpha$, is given by the following observation:

Observation 6.2. For any $0 \leq k \leq 1 / 2$, the $k \alpha$ coefficients of highest degree of $F_{\alpha}(z)$ approach 1, for sufficiently large $\alpha$.

This result follows directly from Inequality (6) and is interesting, for, while we have not found a completely flat roller-coaster polynomial on the last $\alpha / 2$ terms (which would prove the conjecture via Theorem 3.3), we have found a polynomial that comes arbitrarily close to being flat on any portion less than $\alpha / 2$. Notice also that for a given $\alpha$, the coefficients of the almost flat polynomial strictly increase as the degree of the terms increases. Surprisingly, we can create an almost flat polynomial that strictly decreases on the coefficients from the $\lceil\alpha / 2\rceil$-degree term to the $\alpha$-degree term. Such a polynomial may be created by starting with the graph $K_{1} \amalg K_{1}$, which has independence polynomial

$1+2 z+z^{2}$, and then iteratively multiplying by $\frac{1}{2}(1+z)$ and adding $\frac{1}{2} z^{\alpha+1}$ (recall the almost flat roller-coaster polynomial $S\left(F_{\alpha}, z\right)$ is constructed in the same way, except the iteration starts with 1 instead of $\left.1+2 z+z^{2}\right)$.

One possible further area for future work would be computing independence polynomials that result from well-coveredness preserving graph operations. A number of graph 
operations that preserve well-coveredness under certain conditions may be found in [8], including the corona, the conjunction, the disjunction, and the Cartesian product of graphs. The case of the lexicographical product (also called graph composition or the wreath product) has already been solved: it is shown in [8] that the lexicographic product preserves well-coveredness under certain conditions, and in [3], a short proof that the independence polynomial for the lexicographic product $G[H]$ may be directly computed in terms of the independence polynomials for $G$ and $H$ is given.

\section{Acknowledgments}

This research was done at the University of Minnesota Duluth Research Experience for Undergraduates (REU). I would like to give special thanks to Joseph A. Gallian for his encouragement and support, and I like to thank Mike Develin and Stephen Hartke for their insightful comments. I would also like to thank Dan Isaksen and David Moulton for helpful conversations. Finally, I would like to thank the anonymous referee for useful comments on the final draft. Funding for the University of Minnesota Duluth REU was

provided by grants from the NSF and NSA, grant numbers NSF/DMS 9820179 and NSA MDA 904-00-10026.

\section{References}

[1] Y. Alavi, P.J. Malde, A.J. Schwenk, and P. Erdős, The vertex independence sequence of a graph is not constrained, Congr. Numer., 58 (1987), 15-23.

[2] J.I. Brown, K. Dilcher, and R.J. Nowakowski, Roots of independence polynomials of well covered graphs, J. Algebraic Combin., 11 (2000), 197-210.

[3] J. I. Brown, C. A. Hickman, R.J. Nowakowski, The independence fractal of a graph, J. Combin. Theory Ser. B 87 (2003), 209-230.

[4] W. Feller, Introduction to Probability Theory and Its Applications, volume 1, 3rd edition, John Wiley \& Sons, Inc., New York, 1968.

[5] V.E. Levit, E. Mandrescu, On well-covered trees with unimodal independence polynomials, Proceedings of the Thirty-third Southeastern International Conference on Combinatorics, Graph Theory and Computing (Boca Raton, FL, 2002), Congr. Numer. 159 (2002), 193-202.

[6] T.S. Michael, W.N. Traves, Independence sequences of well-covered graphs: Nonunimodality and the roller-coaster conjecture, Graphs Combin., 19 (2003), 403-411.

[7] M.D. Plumber, Some covering concepts in graphs, J. Combin. Theory, 8 (1970), 91-98.

[8] J. Topp, L. Volkmann, On the well-coveredness of products of graphs, Ars Combin. 33 (1992), 199-215. 